\title{
Łukasz Komsta, Yvan Vander Heyden and Joseph Sherma (Eds): Chemometrics in Chromatography
}

\author{
Peter Myers ${ }^{1}$
}

๑) Springer-Verlag GmbH Germany, part of Springer Nature 2019

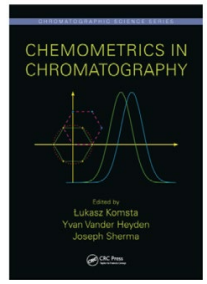

Bibliography

Chemometrics In Chromatography

Łukasz Komsta, Yvan Vander Heyden and Joseph Sherma (Eds)

Chromatographic Science Series

CRC Press, Boca Raton, FL, USA, 2018, pp 506

ISBN 13:978-1-4987-7253-2

GBP 160.00, USD 168.00

Looking for a good read on holiday, well this is not the book but it is one you should have if you are doing any serious method development in separation science. However, in saying that it may be a book that is out of a chromatographer's comfort zone, I say this as over the years I have seen many papers/presentations where there has been a complete disregard to any experimental design in method development. The most I normally see in terms of chromatography theory is the van Deemter equation and it is very rare to see method development using simple graphs of ln $\mathrm{k}$ vs. \% mobile phase.

So, buy this book; it may be a struggle, you may not understand all of it but use it as a textbook and pick and choose the sections/chapters that most relate to your work when your understanding of the theory of chromatography will be enhanced. Or if you just want to improve your method development and method robustness again this book will vastly improve these very important parameters.

So why is this book a must purchase? It consists of seven sections, sub-divided into a number of chapters. Just the first three sections make this a book to purchase. I know many reading this review may say yes "I have done experimental design" and I am sure you have, but after reading just the first section you will gain new insights into what you have been doing and what you should be doing. Similarly, with the

Peter Myers

Peter.Myers@liverpool.ac.uk

1 Department of Chemistry, University of Liverpool,

Liverpool, UK second section on univariate analysis. Once you have read the section on statistics in validation of quantitative chromatography methods you may, like I did, start to worry about a lot of your previous data and consider the data you are obtaining from the manufacturer's print-outs for your chromatograms. Section 3 deals with data processing and unsupervised analysis. I found the chapters in this section difficult to understand, but I stuck with them and I am now pleased that I did as I now know about matrix factorisations and gained a better background for the later chapters. How many times have you heard chromatographers talk about peak shapes as a very simple term? Read Chapter 12 and then you will, like I did, understand about peak shape and talk about peak shapes in a very different light. The only weak chapter for me was Chapter 17 on quantitative structure/retention relationships, but only weak because the others were so good. I have always thought the simple approach of QSRR is not the correct way but read the chapter and make up your own mind. Section 6 gave me some very interesting new insights on herbal fingerprinting. The chapter on chromometric strategies in chromatographic analysis of pharmaceuticals has an excellent reference section. The final section brings together some very interesting bits-and-pieces, for example what about applying Bayesian methods into chromatographic science-ever done this? Well after reading Chapter 22 you may want too.

In conclusion, this is a great book for all chromatographers. It will make you look and consider your experimental designs and the data you collect. Some of the methods described are not standard in your chromatographic data system. But with today's high-performance computers and the vast amount of open source software I am sure the average lab scientist can easily implement the well-described methods in the book.

Publisher's Note Springer Nature remains neutral with regard to jurisdictional claims in published maps and institutional affiliations. 